\title{
Supercontinuum radiation in fluorescence microscopy and biomedical imaging applications
}

\author{
Chetan Poudel ${ }^{1}$ and Clemens F. Kaminski ${ }^{1, *}$ \\ ${ }^{1}$ Department of Chemical Engineering and Biotechnology, University of Cambridge, United Kingdom. \\ *Corresponding author: cfk23@cam.ac.uk \\ Compiled December 24, 2018
}

\begin{abstract}
Compact, high brightness supercontinuum sources have already made a big impact in the fields of fluorescence microscopy and other biomedical imaging techniques, such as OCT and CARS. In this review, we provide a brief overview on the generation and properties of supercontinuum radiation for imaging applications. We review specific uses of supercontinuum sources and their potential for imaging, but also their limitations and caveats. We conclude with a review of recent advances in UV supercontinuum generation, near-IR microscopy, exciting new potentials for the use of hollow core PCFs, on-chip supercontinuum generation and technologies to improve supercontinuum stability for certain applications.

(C) 2018 Optical Society of America
\end{abstract}

http://dx.doi.org/10.1364/ao.XX.XXXXXX

\section{INTRODUCTION}

Fluorescence microscopy and biomedical imaging technologies have made enormous progress over the past two decades and an entirely new set of tools are available in the life sciences that permit targeted structural and functional information to be gained from biological systems. Exciting developments include super-resolution methods [1-4] that go beyond the diffraction limit in optical imaging as well as methods that allow one to measure spectroscopic parameters, such as wavelength spectrum and fluorescence lifetime [5]. These techniques provide information not only on the location of sub-cellular structures but also on their function and environment. The field has been massively boosted by huge technological developments in detectors and light sources, but also molecular labeling methods to permit specific staining of many different targets. The increasing sophistication of bioimaging experiments has placed an ever increasing demand on the enabling photonics technologies. One of the most groundbreaking developments over the past two decades in this context is the compact fiber-based supercontinuum (SC) source.

Exhaustive reviews on the topic of generating SC and its subsequent properties already exist in the literature, for which we refer the reader to [6-11]. The freedom in design afforded by photonic crystal fibers (in tuning hole diameter, periodicity, core size and material) has allowed scientists to study and use the interplay between dispersion, nonlinearity, optical losses and polarization effects [9] to generate tailored SC properties for various applications. Additionally, a number of waveguiding mechanisms can be exploited - using total internal reflection in most solid core fibers, the photonic bandgap effect in hollow core fibers [12], and anti-resonance in some recently reported hollow fiber designs $[13,14]$. SC sources are often (erroneously) referred to as 'white light lasers'. Temporally coherent and laser-like SC can be generated through short pulse pumping, but many of the most useful SC sources for biological applications come from high power, incoherent supercontinua, that are driven by longer pump pulses. Both types of SC radiation can provide high spatial coherence as they are usually generated by the fundamental mode of optical fibers. Average output powers of multiple Watts are routinely achieved in commercial devices, making them suitable for many applications. SC sources have been widely employed in applications ranging from studies of fundamental processes [11] in physics, biology and chemistry through absorption and excitation spectroscopy [15-18]; generating ultrashort femtosecond pulses to create optical clocks and frequency combs [19] for precision frequency metrology (leading to a Nobel prize in 2005); optical communication; atmospheric science, and light detection and ranging (LIDAR) [20]; biomedical imaging using optical coherence tomography (OCT) [21] and coherent anti-Stokes Raman scattering (CARS) [22]; and live cell imaging using various microscopy techniques [23]. In this short review, we present aspects of SC sources most relevant for the demands in microscopy (mostly fluorescence based modalities) and for biomedical imaging applications. We begin with the properties of SC that are critical in the context of imaging. We then provide an overview of methodologies used to select desired wavelengths, and go on to review the wide variety of SC-enabled microscopies reported so far, which include wide- 
field and laser scanning techniques, spectral imaging, lifetime imaging, two-photon absorption, super-resolution and OCT and CARS microscopies. We conclude with an outlook of current challenges in the field, and recent developments that may enable new applications in the future, including the use of gas-filled hollow-core fibers to generate tunable UV radiation with high pulse energies and peak powers.

\section{PROPERTIES OF SUPERCONTINUUM TAILORED FOR IMAGING}

Ranka's[25] use of solid core PCF and a mode-locked Ti:sapphire laser, and Birks's report [26] on tapered optical fibers were the first demonstrations to herald the promise of efficient, costeffective and routine generation of octave-spanning SC in the laboratory. The creation of endlessly single-mode PCFs supporting all generated wavelengths within one fundamental guided mode of the fiber [27] complemented these developments. Today, after over forty years of theoretical and experimental research into SC generation, the spectral broadening mechanisms are understood and have been identified to be soliton dynamics, self-phase modulation (SPM), four-wave mixing (FWM), modulation instability (MI), Raman self-frequency shift (RSFS) and dispersive wave generation (DW) [11, 28-30]. SC can today be generated routinely using a range of fiber types, input pump sources, pulse energies, and input pulse durations ranging from femtoseconds (fs) to continuous wave sources [7]. SC generated by pumping PCFs can be broadly categorized according to the injected pulse durations, as A: ultrafast fs pulses and B: long pulses (ps-ns pulses or continuous wave), summarized in Table 1. This can be further subdivided based on whether the SC generating medium is pumped in the normal dispersion (NDi) or anomalous dispersion (ADi) regimes. The dispersion regimes dictate propagation dynamics and SC generation mechanisms inside the nonlinear fibers. Therefore, choosing the right regime for desired output characteristics is critical, affecting not just the obtainable bandwidth and pulse durations but also properties such as the SC dynamics like coherence and shot-to-shot stability. In what follows, we discuss how these properties (spectral bandwidth, brightness, pulse energy, pulse duration, average power, coherence, stability) can be controlled by selecting appropriate input pulses and dispersion regimes for specific applications, such as fluorescence microscopy.

SC generation over the last few decades was driven primarily by a push to increase spectral bandwidth and create octavespanning SC encompassing the full visible range and NIR. An efficient way of generating such broadband SC is by using fs pulses from Ti:Sapphire (800nm) lasers to pump tailored PCFs. These PCFs are fabricated specifically to place the input source wavelengths in the ADi regime, as in Ranka's experiment [25]. Owing to its enormous potential in optical imaging, this method was picked up already in 2004 by various biophotonics laboratories, reporting either the use of custom-built PCFs [31-33] or tapered silica fibers [34] in the ADi regime with hundreds of $\mathrm{mW}$ input power from $\sim 80 \mathrm{MHz}$ Ti:Sapphire lasers. SC covered a broad wavelength spectrum from $\sim 400 \mathrm{~nm}$ to near IR wavelengths of $\sim 1000-1500 \mathrm{~nm}$ because of efficient broadening via soliton dynamics. The spectral power densities were fairly low (less than $0.5 \mathrm{~mW} / \mathrm{nm}$ ) but usable for general fluorescence microscopy applications.

However, pumping in the ADi regime also leads to high instability arising from shot noise in the input laser pulses, which gets amplified stochastically by modulation instabilities [7]. This leads to temporally incoherent SC light with uneven spectra and large differences in spectral density profiles from shot-to-shot (see Figure 1). SC instability does not necessarily pose a problem in general fluorescence microscopy applications since the timescale of experiments is much longer than the instantaneous fluctuations, causing them to be averaged over. Temporally incoherent light is, in fact, often favored for imaging because coherent waves can introduce unwanted speckle patterns in the image. On the other hand, applications such as CARS need a high degree of coherence, for which fs pumping of all-NDi fibers is more suitable since modulation instability and soliton-related effects do not occur in this case. This generates stable, flat and coherent SC with much better signal-to-noise [24, 35], albeit with smaller spectral widths at comparable peak power [36].

A big limitation in using fs pumps is the maximum obtainable SC power. The peak intensity damage threshold in microstructured fibers usually limits the maximal spectral power density to $\sim 0.5 \mathrm{~mW} / \mathrm{nm}$. For applications requiring higher power densities, using ps-ns input pulses increases it to several $\mathrm{mW} / \mathrm{nm}$ in the visible range [37]. Of course, when longer pulses are used as input pump sources, the instability problem is greater (both in the NDi and ADi regimes): larger fluctuations are seen, and the coherence of the SC is compromised. Despite the stability issue, the massive development of fiber laser sources over the last few decades has provided cost-effective, reliable means of providing ps-ns input pulses for SC generation with low maintenance and smaller fingerprint than fs sources. Therefore, for microscopy and bioimaging applications, generation of SC has mostly switched over to using these ps-ns fiber laser pump sources. The technology has also been commercialized by a few companies making SC generation widely accessible through a variety of compact turnkey sources, usable by general biology laboratories [22]. Further efforts over the last decade have resulted in SC sources with higher power, faster pulses and greater range of wavelengths. As an example of commercial technology, one of the widely successful incoherent SC source is based on coupling ps pulses from a mode-locked (typically $40 \mathrm{MHz}$ ) ytterbium fiber laser into an engineered PCF with a ZDW around $1050 \mathrm{~nm}$, yielding high average SC output powers at up to $\sim 20 \mathrm{~W}$ with 6ps pulse widths, few hundred nJ maximum pulse energy and single-mode operation in the 400-2400nm range [38]. Groups working on higher average output from ps sources have reached powers up to $39 \mathrm{~W}$, with $31.7 \mathrm{~mW} / \mathrm{nm}$ spectral power density and good uniformity across the full visible spectral range [39].

SC has also been generated using continuous wave sources like fiber lasers using simple, cost-effective setups yielding broad spectral profiles. Massive output power densities (10s of $\mathrm{mW} / \mathrm{nm}$ ) can be generated from 5-50W pump sources and used in applications where high average brightness is more important than short-pulse characteristics, e.g. peak power. However, SC generated from $\mathrm{CW}$ input undergoes significant intensity fluctuations and has negligible temporal coherence due to modulation instability [7]. The spectral coverage usually lies in the NIR with bandwidth not large enough to extend into the visible range. This is one of the biggest limitations of CW input for SC generation as obtaining output in the visible spectrum is of prime importance in applications like fluorescence microscopy. Some attempts have been made to increase bandwidth and simultaneously push it towards the visible range going down to $600 \mathrm{~nm}$ [40] but this required industrial class fiber lasers requiring enormous pump powers of $400 \mathrm{~W}$, which is impractical for routine use. Another concept for extending to shorter wavelengths in 

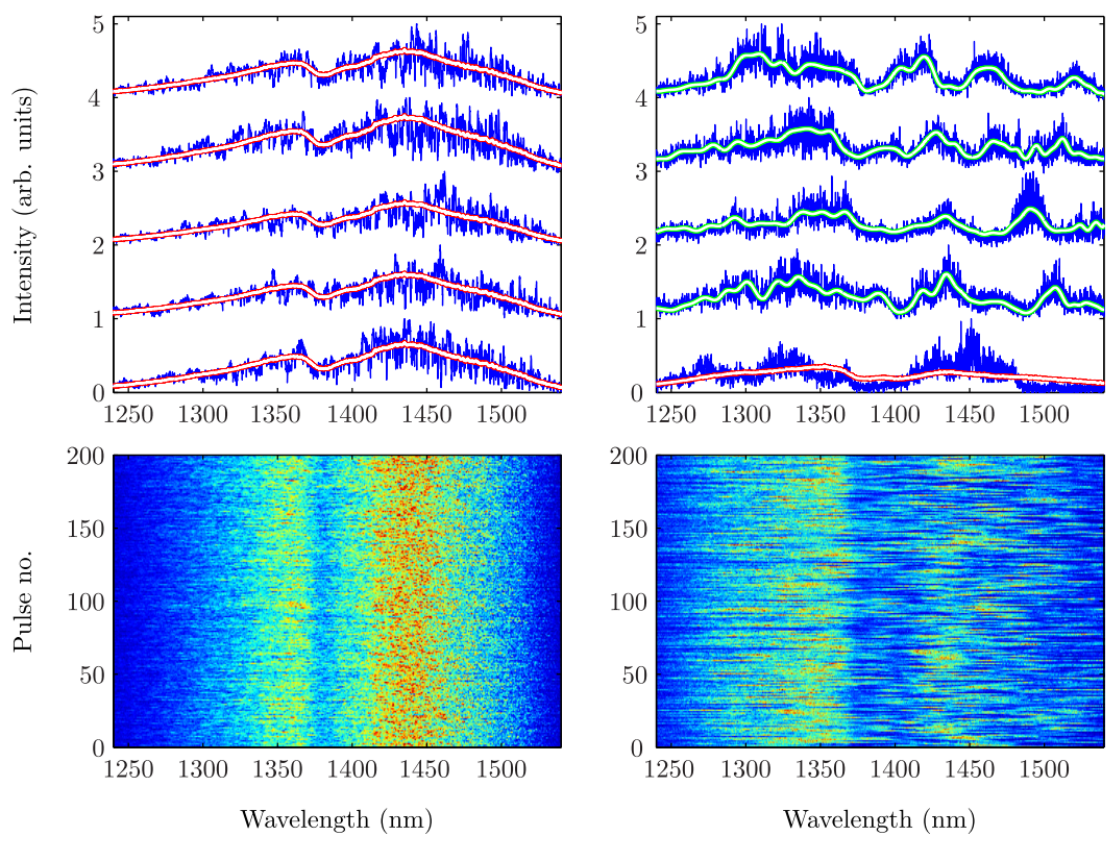

Fig. 1. Comparison of shot-to-shot differences in SC spectra obtained with femtosecond pumping in the normal dispersion (NDi - left panels) and anomalous dispersion regimes (ADi - right panels). The red/white lines (top left) represent a long term average $(10,000$ shots) of the all-NDi spectrum, where each individual shot trace (blue) mimics the shape of the average long-term spectrum. This stability in the NDi regime can also be observed in the 200 consecutive single-shot spectra (bottom left). The green/white lines (top right) are low pass filtered versions of individual shot-to-shot spectra, all of which show significant deviations from their long term average (red/white line in the bottom) in the ADi regime SC. Consecutive single shot spectra also show large differences (bottom right) in the ADi regime. Clearly, the dynamics of SC in the NDi regime are in stark contrast with dynamics in the ADi regime. Adapted from [24].

\begin{tabular}{|c|c|c|}
\hline Pulse duration & Femtosecond pulses & Pico-nanosecond pulses and continuous waves \\
\hline Spectral width & Broad spectrum (visible-NIR) & $\begin{array}{l}\text { Broad spectrum when using pulsed input, some covering } \\
\text { 400-2400nm; Mostly only IR spectrum for CW sources }\end{array}$ \\
\hline $\begin{array}{l}\text { Coherence and } \\
\text { stability }\end{array}$ & $\begin{array}{l}\text { Coherent and stable (NDi regime); } \\
\text { Incoherent, unstable (ADi regime) }\end{array}$ & $\begin{array}{l}\text { Usually incoherent, unstable but can be coherent with ps } \\
\text { sources (NDi regime); Large fluctuations, incoherent for CW }\end{array}$ \\
\hline $\begin{array}{l}\text { Spectral power } \\
\text { density }\end{array}$ & Low, around $0.5 \mathrm{~mW} / \mathrm{nm}$ & $\begin{array}{l}\text { Moderate values for ps-ns pulses, around few } \mathrm{mW} / \mathrm{nm} \text {; } \\
\text { High power for CW: } 10 \mathrm{~s} \text { of } \mathrm{mW} / \mathrm{nm}\end{array}$ \\
\hline $\begin{array}{l}\text { Pump peak } \\
\text { power }\end{array}$ & fs input: few $\mathrm{kW}$ & ps-ns input: Usually 100s of W; CW input: Few W \\
\hline Convenience & $\begin{array}{l}\text { Usually very expensive, complex, } \\
\text { and difficult to maintain }\end{array}$ & $\begin{array}{l}\text { Cost-effective and low maintenance. Widespread applica- } \\
\text { tions for pulsed output. }\end{array}$ \\
\hline
\end{tabular}

Table 1. General characteristics of different SC generated by altering pump sources.

CW SC was demonstrated through fiber tapering, causing dispersive waves to be further blue-shifted. Wavelength extending to $650 \mathrm{~nm}$ can thus be reached even with moderate pump powers of around 35W [41]. Finally, through Ge-doping and fiber tapering, the first pure CW white-light SC was generated in 2012 with a spectrum spanning $470 \mathrm{~nm}$ to more than $1750 \mathrm{~nm}$ at $9.3 \mathrm{~W}$ power [42].

\section{WAVELENGTH SELECTION SCHEMES}

Perhaps the most useful property of a SC source in fluorescence microscopy lies in its massively broadband wavelength spec- trum. Traditionally, fluorescence studies of cellular processes have used mercury and xenon arc lamps for broadband illumination but suffer from low illumination efficiency and low spatial coherence, and therefore cannot be used to improve resolution in scanning-microscopy applications. Diodes and monochromatic lasers provide bright illumination without the necessary broad bandwidth. The handful of monochromatic lasers that are commonly available pose an unnecessary restriction on the vast available toolbox of excitable fluorophores, rendering only a few fluorophores usable whose excitation wavelengths match the fixed laser wavelengths. Even then, the most efficient excitation 
a

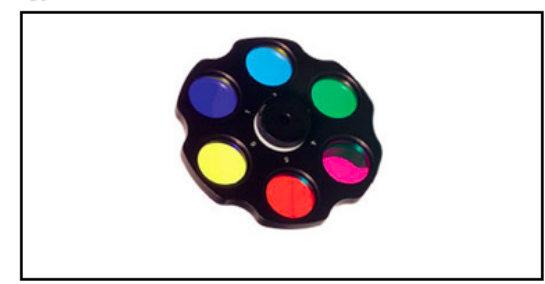

Filter wheel

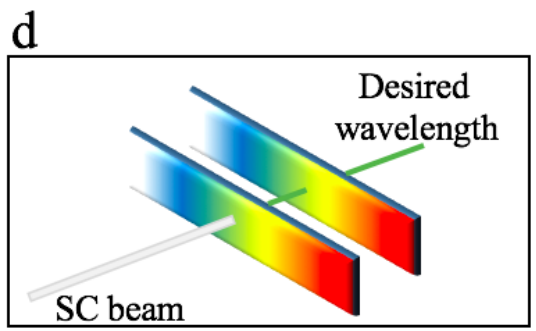

LVTF b

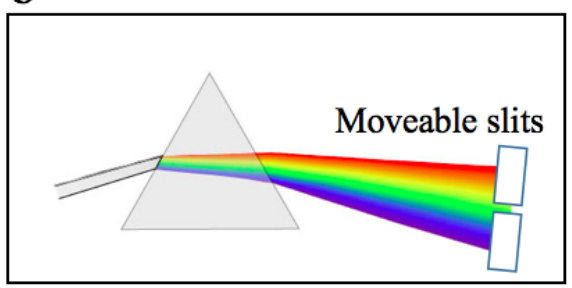

$\underline{\text { Prism }}$

e

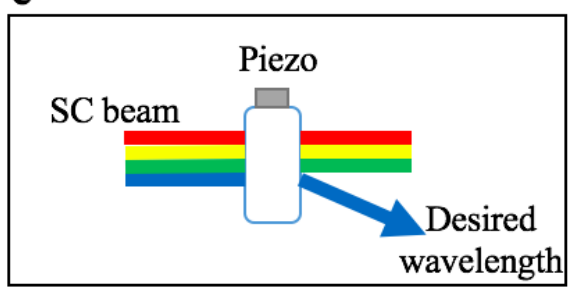

$\underline{\mathrm{AOTF}}$
C

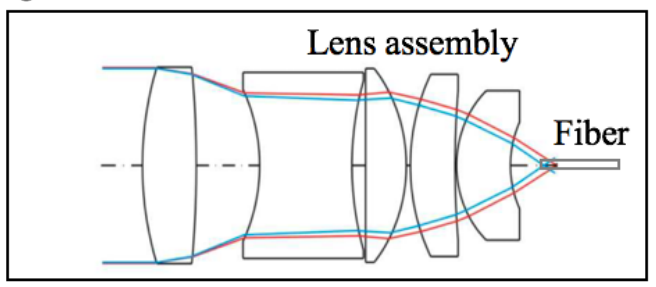

Axial monochromator

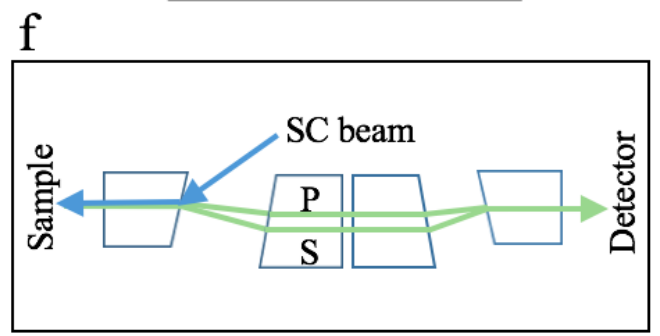

AOBS

Fig. 2. Different schemes to select desired excitation bands from SC sources: (a) filter wheel (b) prism [32] (c) axial monochromator [43] (d) LVTF [44] (e) AOTF [45] and (f) AOBS [46]. Their individual characteristics are summarized in Table 2.

\begin{tabular}{lcccccc}
\hline \hline Characteristics & Filter wheel & Prism & Axial monochromator & LVTF & AOTF & AOBS \\
\hline High transmission & + & - & + & + & - & + \\
Tuning speed & - & - & - & + & + & + \\
Wide wavelength range & + & + & + & + & + & + \\
Tunable bandwidth & - & + & - & + & - & - \\
Simultaneous multi-color & - & - & + & - & + & + \\
Out-of-band suppression & + & + & - & - & - & - \\
Steep spectral edge & + & - & - & - & - & - \\
Polarization insensitive & + & - & - & + & - & + \\
\hline
\end{tabular}

Table 2. Wavelength selection schemes with their pros(+) and cons(-).

wavelength can lie between available laser wavelengths [23].

The increasing complexity of bioimaging experiments and rising demand to simultaneously image in multiple colors necessitate flexible use of excitation wavelengths across the entire visible range to avoid crosstalk. Previously, dye laser systems with tunable wavelengths have been used [47] to excite multiple fluorophores, but the gain curves of these dyes limit the tuning range to $50-100 \mathrm{~nm}$ and do not cover a significant portion of the visible spectrum. Tunable Ti:Sapphire lasers provide another alternative to access the full visible spectrum but they can only achieve this through processes like multi-photon absorption, harmonic generation and optical parametric oscillators (OPO), making the process expensive and difficult to operate, and requiring specialist maintenance. SC sources overcome most of these spectral restrictions with a large bandwidth spanning the visible and NIR without any gaps, and thus lifting the restrictions of matching fluorophores to available laser lines. The full SC spectrum is rarely used simultaneously for imaging. Most fluorescence applications pick out desired excitation wavelength bands from the output. While this means that most of the power is discarded, the average power of the spectrally selected out- put ( $\sim 1 \mathrm{~mW} / \mathrm{nm}$ from commercial sources) is still sufficient for most imaging applications [32]. In conjunction with versatile wavelength selection schemes, a SC source can simultaneously excite a number of fluorophores, each at their optimal absorption wavelengths. This provides high specificity with minimal cross-excitation and permits studies of various structures and phenomena at the same time.

Good wavelength selection schemes should provide full flexibility in selecting wavelengths and bandwidth over a large spectrum at high speed with potentials for multiplexed imaging. Many such technologies have been proposed but the most noteworthy ones (see Figure 2) include bandpass filter wheels, prism-based spectrometers [32], axial monochromators [43], motorized linear variable tunable filters (LVTF) [44], acousto-optic tunable filters and beam splitters (AOTF and AOBS) $[45,46]$. In Table 2, we summarize and compare the characteristics of these technologies.

Using a filter wheel with 6-12 bandpass filters can accommodate a wide range of wavelengths. The high-precision multilayer coatings on these filters provide excellent out-of-band suppression (usually over OD 5) and high edge steepness. How- 
ever, changing wavelengths requires physical movement of the filter wheel in the beam path over 50-200ms timescale (when motorized), precluding the possibility of simultaneous multiwavelength selective excitation. Dual, triple or quad band filters are now available for performing simultaneous excitation but these are not tunable for arbitrary selection. A different tunable implementation uses prisms to spatially disperse the beam, part of which goes through a translatable aperture to select a central wavelength and bandwidth [32], but this leads to large power losses and does not allow simultaneous excitation of multiple wavelength bands. Another excitation technique uses an on-axis monochromator based on a custom-designed lens to intentionally maximize the beam's longitudinal chromatic aberration while keeping other aberrations low [43]. This wavelength-dependent longitudinal dispersion of foci along the optical axis allows coupling desired part of the focused beam into a finite aperture fiber and discarding all other out-of-focus wavelengths.

To continuously tune the central wavelength along with bandwidth, one can use LVTFs whose cutoff wavelengths vary linearly along their length. A useful configuration involves the SC beam going through two LVTFs (one as shortpass, one as longpass) translated independently to make a variable bandwidth filter[44]. Circular LVTFs use the same concept but work by rotating the filters. LVTFs work well for tuning excitation when the optical beam width is small in cross section as finite beam widths deteriorate the edge steepness. The need for mechanical translation slows their tuning speed ( $\sim$ hundreds of $\mathrm{ms}$ ) and they cannot be used for simultaneous wavelength selection.

In contrast to previous technologies, AOTFs provide very fast, programmable wavelength tuning ( $\mu \mathrm{s})$ with simultaneous output possible for up to eight different wavelengths over a large spectral range (hundreds of nanometers) with no moving parts, demonstrated [48] for spectrally-resolved imaging. Acousto-optic technologies effectively produce a phase grating to diffract a specific part of the incident light with very narrow passband $(\sim 1 \mathrm{~nm})$ under phase-matching conditions of optical and acoustic waves. However, AOTFs do suffer from low out-ofband suppression, low transmission due to polarization selectivity, and sometimes from wavelength sidebands that introduce a wavelength dependent angular spread in their output [48], which degrades image quality. Additional compensation optics like prism elements can correct for polarization selectivity to restore high transmission and remove angular spread, and have been commercialized as acousto-optic beam splitters (AOBS) [34, 46], making them a single versatile instrument handling both excitation and emission selection efficiently.

No single wavelength filtering scheme fulfills the needs of all imaging experiments, although acousto-optic technologies provide more versatility than others. It is important to evaluate and choose wisely between them by considering the criteria (eg. speed, tunability, out-of-band suppression to minimize crosstalk, simultaneous multi-color) most relevant for the experiment in question. Once spectral selection is performed appropriately, the output light can be coupled to a microscope directly for widefield imaging, to a scan unit for point-scanning, or modified in other ways (eg. beam shaping, pulse compression) for desired applications.

\section{MICROSCOPY MODALITIES USING SUPER- CONTINUUM RADIATION}

SC sources have revolutionized microscopy for biological and medical imaging applications, offering significant advantages over monochromatic lasers in spectral flexibility and over traditional lamp sources in terms of fast pulsed nature, high brightness, spatial coherence, deep tissue penetration and contrast [50], low-maintenance, and cost-effectiveness. In what follows, we discuss how SC properties are exploited in different microscopy applications. We review the use of SC spectral flexibility, particularly in hyperspectral imaging; SC spatial coherence used for point scanning microscopies; the fast pulsed nature of SC for spectroscopy and fluorescence lifetime imaging (FLIM); high peak power applications in multi-photon excitation and second harmonic generation; simplification of super-resolution microscopy instrumentation using SC sources; and finally, the bright, coherent light applications of SC for OCT and CARS microscopies.

\section{A. Widefield and confocal scanning techniques, and wavelength-resolved imaging}

SC sources provide a wider spectrum of wavelengths and much greater brightness than thermal sources or LEDs for widefield fluorescence microscopy techniques. In particular, incoherent $\mathrm{SC}$ is best for widefield imaging since it provides flat-field illumination without generation of speckle artefacts or aberrations. These artefacts are seen with traditional coherent laser sources due to interference between light waves and degrade image quality $[51,52]$. Incoherent SC with large spectral bandwidth and excellent beam profiles are marketed commercially, permitting easy integration into traditional setups for widefield microscopy. The SC output can additionally be launched into a multimode fiber to impose a strong spatial incoherence and uniform illumination field. SC sources have found a greater market in confocal laser scanning fluorescence microscopy, which was the first bioimaging application to adopt SC radiation [31, 32]. This is because fiber-generated SC radiation can easily be focused onto a diffraction-limited spot and point-scanned through the sample. It is conceivable that high power commercial SC sources will replace monochromatic lasers in all future confocal microscopes, simplifying the setup, reducing costs and enhancing versatility [37]. It is important to consider the effect of longitudinal chromatic aberration and chromatic variations in beam divergence potentially affecting the spatial resolution. A study quantifying and comparing 3D point spread functions (PSF) in the blue and red spectral regions in a confocal setup utilizing a commercially available SC source found that the displacement of the focal spot along the $\mathrm{z}$-axis was comparable in extent to the full-width-half-maximum of the PSF [48]. Therefore, chromatic aberrations caused by using a SC source do not pose a significant limitation for performing high-resolution confocal imaging in multi-color throughout the visible spectrum. In other techniques like volumetric confocal reflectance microscopy [53], this chromatic aberration feature has been maximized using aspheric lenses because it permits one to encode depth information spectrally and to be read out by a spectrum analyzer or spectrometer. This way, multiple depths in biological specimen can be probed simultaneously and rapidly, with one group demonstrating a $157 \mu \mathrm{m}$ axial range with micrometer resolution captured in a single shot from epithelial tissue [54]. This technique eliminates the need for mechanical axial scanning, making it useful even for endoscopic imaging systems. 

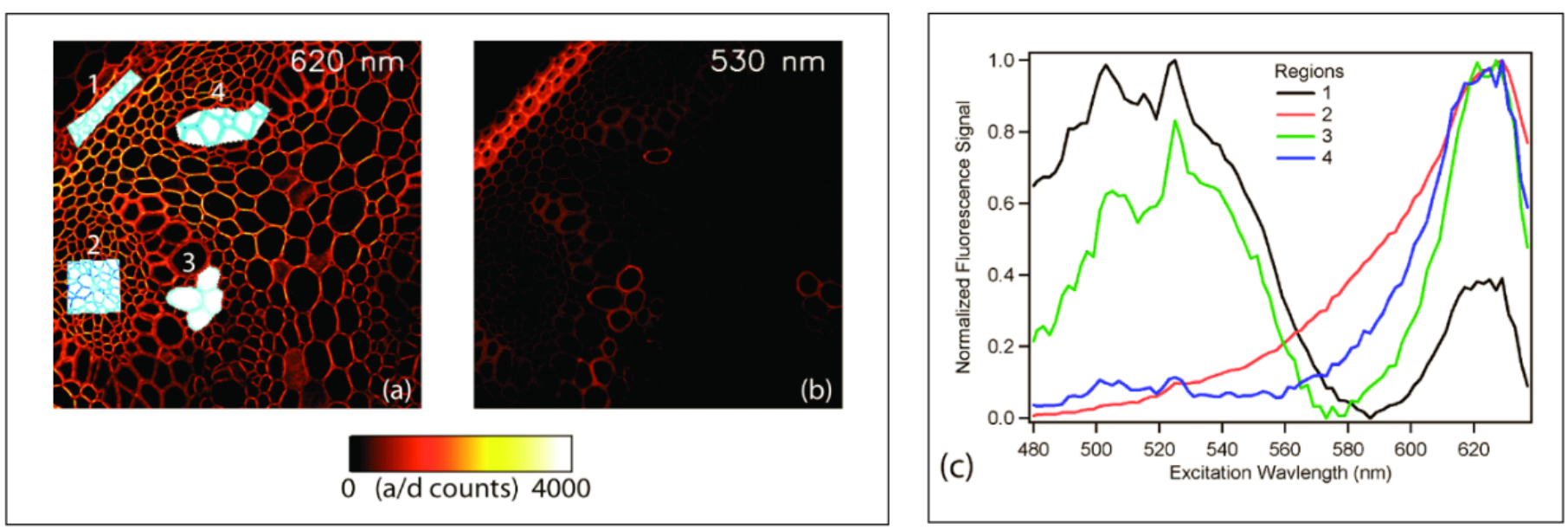

Fig. 3. Hyperspectral imaging reveals that different regions of Convallaria majalis have different emission properties at (a) 620nm and (b) 530nm excitations. (c) Excitation-emission plots for four regions in (a). Adapted from [49].

Combining the flexible excitation from a SC with appropriately multiplexed emission detectors enables the method of hyperspectral imaging (wavelength-resolved imaging over a continuous spectral range, see Figure 3). Hyperspectral imaging allows capturing and completely characterizing the entire excitation and emission profile of known and unknown fluorophores and biological samples at all available wavelengths for each pixel of the image $[23,49,55,56]$. This information can be used in post-acquisition spectral unmixing methods to reliably discriminate fluorophores with overlapping spectra in biological studies $[57,58]$. The same technique can be used to remove unwanted autofluorescence in the emission channel that might be affecting the data. In other cases, tissue autofluorescence may contain useful information. Tissues undergoing disease progression can show structural and metabolic changes affecting their absorption, scattering and fluorescence characteristics [59] and these subtle changes can be picked up by SC-based hyperspectral imaging techniques. This has been particularly successful in label-free imaging of biological specimens. Probing for more spectral information using SC at extended wavelengths and increased acquisition rates will greatly enhance non-invasive disease diagnostics [60].

\section{B. Fluorescence lifetime microscopy (FLIM) and multi- parameter imaging}

The inherent pulsed nature of most SC sources is useful for timeresolved fluorescence spectroscopy. This technique can measure the excitation and emission spectra of fluorophores, along with the average lifetime of their fluorescence decay with a hightemporal (sub-ns) resolution. The fluorescence decay lifetime of fluorophores is exquisitely sensitive to their micro-environment and can provide a great deal of quantitative information about the $\mathrm{pH}$, local viscosity, temperature, ion concentration, protein aggregation, protein binding, and kinetics of chemical reactions around the fluorophores $[5,61]$. SC sources provide the highfrequency $(\sim \mathrm{MHz})$ pulsed excitation required for lifetime measurements and now some commercial sources can also offer easy tuning of this repetition rate $(80,40,20,10,5$, etc. in $\mathrm{MHz})$ for capturing shorter or longer lifetimes. The short pulse width (few ps) of the SC output helps reduce errors in temporal measurements. Selecting narrow bands from the SC spectrum also allows specificity in exciting fluorophores with distinct spectra and life- times at their optimal absorption wavelengths. SC sources can replace fast-pulsed flash lamps in spectrofluorometers [62], providing higher brightness to improve signal-to-noise ratios for sensitive measurements. Merging the capabilities of spectrofluorometry with spatial imaging leads to a powerful quantitative imaging technique called fluorescence lifetime imaging (FLIM). Several implementations of FLIM using SC radiation are in use, including widefield- and spinning-disk-based systems (timegated FLIM $[63,64]$ and frequency domain FLIM [65, 66]), and the more popular point-scanning-based time-correlated singlephoton counting (TCSPC) FLIM systems [32]. All these FLIM implementations can provide the spatial distribution of fluorophores, and their individual percentage contribution to fluorescence in each image pixel.

Like hyperspectral imaging, unmixing multiple fluorophores in an image can be done using fluorescence lifetime as a parameter, even with minimal a priori information [67]. FLIM can also be applied to reveal the intrinsic lifetime contrast (see Figure 4) to investigate health states of tissues. The autofluorescence lifetime characteristics can be studied by simply exciting endogenous fluorophores without labels $[5,61]$ at desired wavelengths using a SC source. SC sources have opened up a more practical way to study Forster resonance energy transfer (FRET) using FLIM to observe complex protein interactions, protein structures and molecular conformations at high-speeds in live cells [68]. By minimizing cross-excitation of acceptors, SC sources enable multi-channel FLIM-FRET [69] from the same laser source. Some advanced FLIM setups using SC can capture multi-parametric and hyperspectral 6D [56] and 7D [55] data including not just the spatial profile and absorption-emission spectra but also simultaneously the fluorescence lifetime and polarization anisotropy profiles $\left(\mathrm{x}, \mathrm{y}, \mathrm{z}, \tau, \lambda_{\text {ex }}, \lambda_{\text {em }}, \mathrm{r}\right)$ for each pixel. This presents great opportunities to improve studies of multiplexed signaling pathways, tissue properties, and functional aspects at the molecular scale.

While the temporal instability of incoherent SC sources may be an issue in certain applications, fluorescence lifetime measurements are mostly unaffected by the shot-to-shot variations because this noise is small compared to the photon-shot noise in imaging systems. In the most widely used TCSPC implementation, only a small fraction of excitation pulses generate a detectable photon to avoid photon pileup effects. On the 

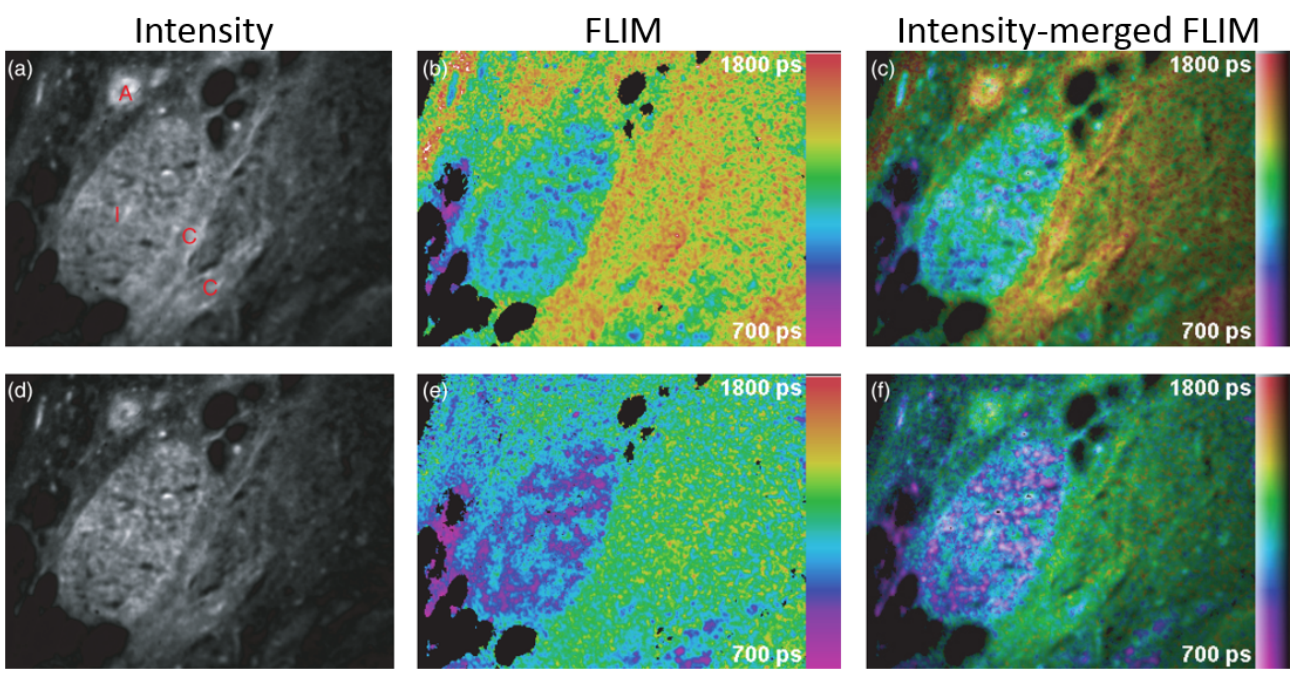

Fig. 4. Intensity and intensity-merged FLIM images of autofluorescence from a section of unstained human pancreas showing clear lifetime differences between tissue types: A-artery, I-islets of Langerhans and C-collagen connective tissue. (a)-(c), $\lambda_{\mathrm{ex}}=440-450 \mathrm{~nm}$, $\lambda_{\mathrm{em}}>470 \mathrm{~nm}$. (d)-(f), $\lambda_{\mathrm{ex}}=520-530 \mathrm{~nm}, \lambda_{\mathrm{em}}>570 \mathrm{~nm}$. Adapted from [32].

timescales of TCSPC FLIM image acquisition (seconds to minutes), the average power of the SC is fairly stable [32]. However, other techniques like fluorescence correlation spectroscopy (FCS) require much more stable lasers and cannot make use of the SC radiation used for most imaging applications, produced by pumping PCFs in the ADi regime. FCS as a technique relies on detecting fluctuations in fluorescence intensity from a small sample volume and therefore, any fluctuations stemming from varying laser illumination will introduce errors in the acquired FCS curve, negatively affecting the experimental outcome [70]. Despite this caveat of instability, other SC features like spectral tunability, simplicity and cost-effectiveness has made the fiberlaser-pumped SC a more attractive option than mode-locked Ti:Sapphire lasers in many FLIM and multi-parametric imaging applications.

\section{Super-resolution microscopy: Stimulated Emission Deple- tion (STED)}

The ability to arbitrarily choose and rapidly change excitation wavelengths using a SC source greatly enhances the number of usable photo-activated dyes and fluorophores [71]. These fluorophores can be turned on and off with different wavelengths and form the basis of many super-resolution techniques like photo-activated localization microscopy (PALM), stochastic optical reconstruction microscopy (STORM) and stimulated emission depletion (STED), among others.

The high illumination densities $\left(\sim 5 \mathrm{~kW} / \mathrm{cm}^{2}\right)$ necessary for PALM and STORM $[3,4]$ require very high laser powers $(\sim 50$ $100 \mathrm{~mW}$ ) at specific wavelengths not attained easily using SC sources. However, STED instrumentation greatly benefits from the simplicity, easy maintenance and affordable cost of a SC source. STED uses one laser pulse focused onto a diffractionlimited spot for fluorophore excitation. Simultaneously or a few ps later, the excitation pulse is followed by a donut shaped redshifted laser pulse to de-excite fluorophores through stimulated emission (see Fig.5). Only a few fluorophores confined in a subdiffraction-sized donut center are allowed to fluoresce, improving the imaging resolution beyond the diffraction limit [1] and reaching resolution below $50 \mathrm{~nm}$. A pulsed tunable SC source used for STED can include wavelength ranges not covered by common pulsed laser sources[72], opening the possibility of using an increased range of dyes [73]. The SC also provides both the excitation and depletion wavelength bands from the same source, eliminating the second laser entirely. Easy and stable temporal alignment can be achieved without the need for synchronization of multiple excitation and depletion sources. SC sources also readily provide ps pulse width output, which are most efficient for STED, obviating the necessity for pulse-stretching of fs range pulses given by Ti:Sapphire lasers. Thus, SC sources allow making major simplifications to the very complicated instrumentation, which is one of the primary drawbacks of STED microscopy. In an example from a few years ago, simultaneous two-color STED was demonstrated for the co-localization of two different proteins at super-resolution $(35 \mathrm{~nm}$ lateral, $90 \mathrm{~nm}$ axial resolution) in 3 dimensions with a single commercial SC source (SC-450-PP-HE) instead of four very-expensive pulsed laser sources that would normally be required [74]. This SC source provided pulse energies (20nJ for 20nm bandwidth at $1 \mathrm{MHz}$ ) comparable to that of lasers commonly used in STED but the acquisition speed was limited by the low repetition rate. Improving resolution at greater speeds with STED depends on very efficient depletion with high pulse powers and therefore will benefit from improved SC sources providing greater pulse powers even at higher repetition rates. However, the main drawback in using SC for STED is the large fluctuation in the pulse energy which can vary from $10 \%$ to $80 \%$ and can induce large optical noise and hinder certain applications[7]. Balanced detection schemes might be able to reduce the noise down to shot noise levels in these cases.

\section{Two-photon excitation (TPE) microscopy and second har- monic generation (SHG)}

Achieving greater peak powers in custom-built SC sources has permitted two-photon excitation (TPE) microscopy with these sources. TPE is caused via the absorption of two photons, each around twice the wavelength of a single photon excitation event $[77,78]$. This allows probing into deeper tissue by using redshifted wavelengths to increase penetration depth and minimize scattering and photodamage. This technique has been valuable in neuroscience and in medical studies involving imaging 


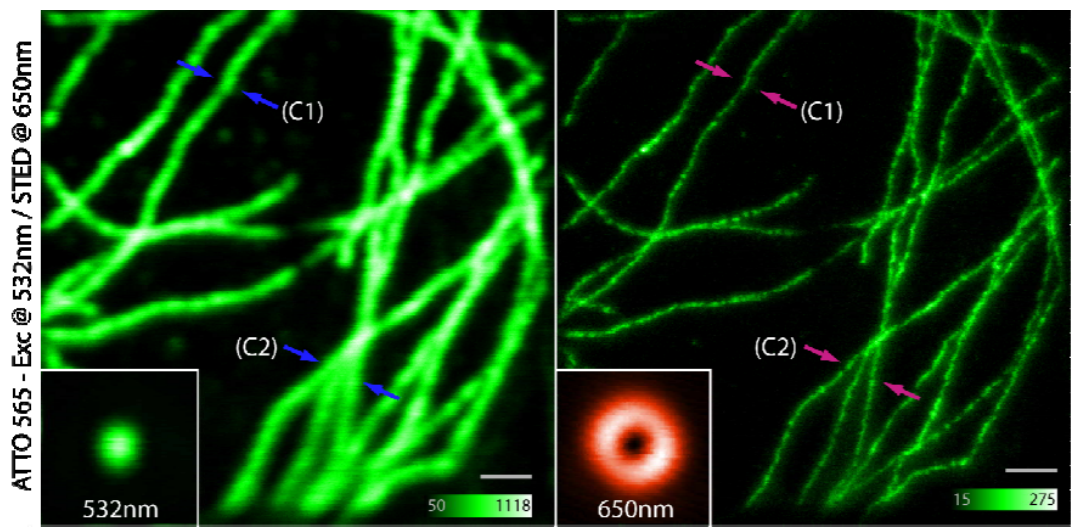

Fig. 5. STED of immunolabeled tubulin fibers with 532nm excitation and 650nm depletion donut beam. Comparing between confocal image (left) and STED (right) shows the resolution enhancement with STED. Adapted from [72].
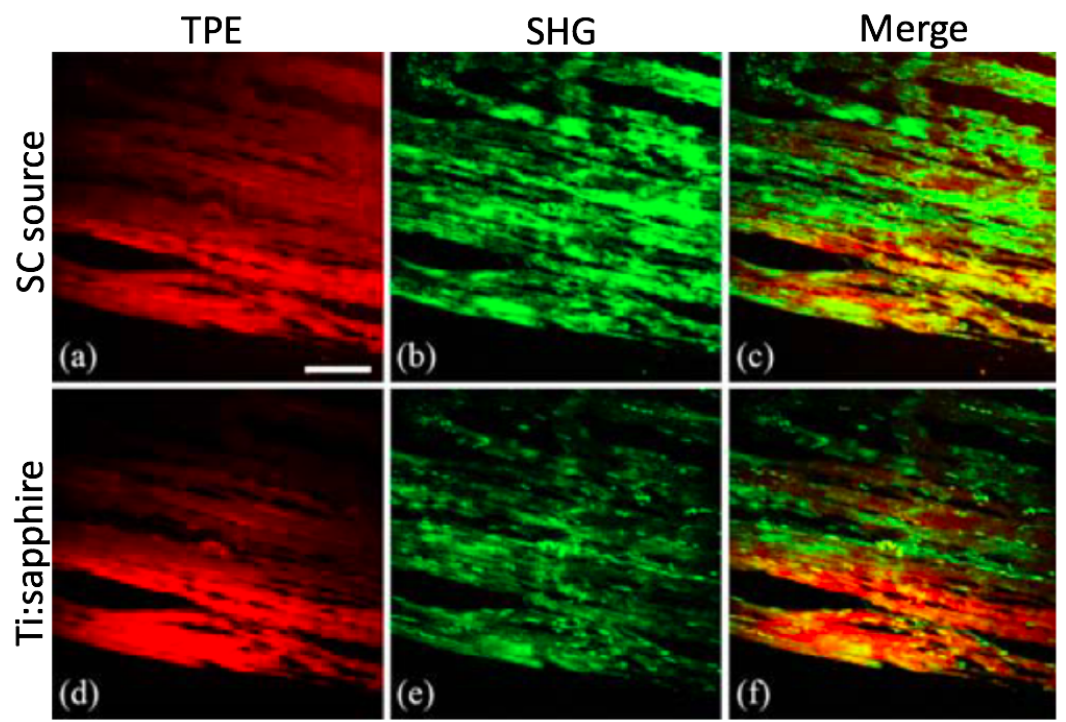

Fig. 6. Two-photon excitation of fluorescence, second harmonic generation and merge images of mouse muscle comparing the performance of a nanosecond SC excitation (a-c) against femtosecond Ti:sapphire excitation (d-f). Adapted from [75].

in-vivo or in thick tissues. However, TPE is a nonlinear process requiring very high photon fluxes in a small confined focal volume (using large peak powers at the sample and typically at least $0.01-0.1 \mathrm{~nJ}$ energies for laser-scanning) in a very short timeframe. It has traditionally only been possible using modelocked femtosecond lasers that compress the high laser power into ultrashort fs pulse packets. By giving out one intense pulse every 10-25ns, these lasers manage to excite two-photon processes while keeping average power into the sample relatively low to avoid photobleaching and phototoxicity. When femtosecond input pulses are pumped through microstructured fibers, in addition to wavelength broadening, they also exhibit dramatic temporal broadening to yield output pulse durations of a few ps, but the pulse energies and peak intensities can still be large enough for TPE.

Two-photon excitation using SC was achieved more than a decade ago by using the near IR part of the spectrum (17W peak power and $1.7 \mathrm{GW} / \mathrm{cm}^{2}$ at the sample, sufficient for TPE even with pulse lengths of 1-5ns) [34]. Others used the visible range of the SC (500-600nm) [33] and used pulse compression techniques to increase peak intensities [79]. Simultaneous three-color
TPE was also demonstrated using fs pulse SC output and $8 \mathrm{~mW}$ power at the sample [80]. However, in all these cases, generating SC with the appropriate characteristics for TPE required pumping PCFs with the fs Ti:Sapphire lasers, making them impractical in most general biology laboratories. The challenge, then, is for longer pulse SC sources to be able to perform TPE efficiently by generating large enough peak powers to achieve the required power densities. In 2016, Lefort et al. [75] demonstrated that both TPE and second harmonic generation (SHG) could be possible from a compact broadband SC source with ns pulses and sufficient peak power. Image quality (see Figure 6) using the SC source (1ns pulses with $370 \mathrm{~nJ}$ energy, $370 \mathrm{~W}$ peak power, $92 \mathrm{~mW}$ average power) was comparable to the Ti:Sapphire (150fs pulses with 70pJ energy, $460 \mathrm{~W}$ peak power, $5.5 \mathrm{~mW}$ average power). Over the years, as novel fibers and ns pump source technologies become mature, TPE should be widely accessible using compact and stable turn-key SC sources that provide large spectral densities with higher peak powers and intensities. 

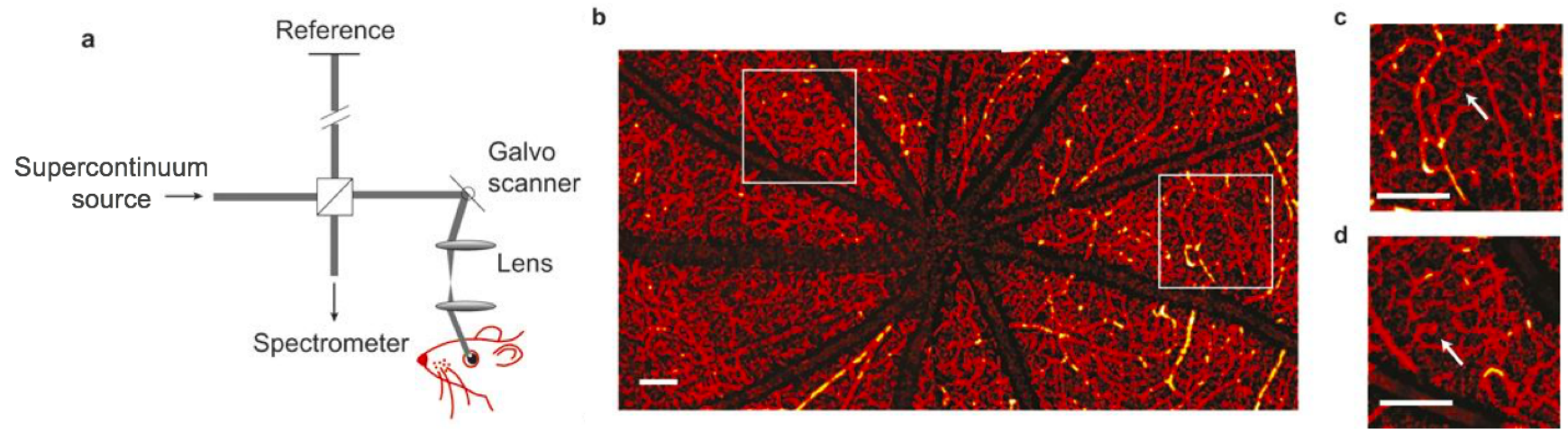

Fig. 7. (a) Schematic of an OCT setup using visible light (520-630nm) from a commercial SC source for in vivo retinal imaging to measure oxygen metabolism. (b) Wide-field view of retinal micro-vasculature imaged using this setup. (c-d) Magnified view of highlighted areas in (b), with arrows showing the smallest capillary vessels. Scale bar: $200 \mu \mathrm{m}$. Adapted from [76].

\section{E. Optical coherence tomography (OCT)}

Optical coherence tomography (OCT) is a non-invasive optical analogue of ultrasound techniques, enabling depth-resolved imaging of biological tissues (see Figure 7) at $\mu \mathrm{m}$ scale resolution by using interferometric detection of photon time of flight [37]. Nowadays, OCT is routinely used in the diagnosis of retinal diseases [81]. OCT ideally requires a stable and noiseless, spatially coherent source with low temporal coherence, single transverse mode and high brightness (power of few $\mathrm{mW} / \mathrm{nm}$ ). The axial resolution of fine microstructures in OCT scales as $\lambda^{2} / \Delta \lambda$, depending inversely on the bandwidth [82], and thus benefits greatly from a source with long range NIR bandwidth. These requirements for OCT are very difficult to fulfill and in the past, super-luminescent diodes and thermal sources were used for their bandwidth to provide $10-15 \mu \mathrm{m}$ axial resolution. However, the low brightness of these sources limits their use in clinical OCT, as higher brightness is necessary to counter signal attenuation in strongly scattering biological tissue and to reduce the integration time in real-time imaging of in-vivo tissues [60].

SC sources fulfill most of the necessary criteria listed above and have been shown to improve resolution by an order of magnitude, while providing higher brightness and faster acquisition in state-of-the-art high-resolution OCT measurements. While resolution can also be improved by using highly specialized broad-bandwidth, ultrashort-pulsed, mode-locked Ti:Sapphire lasers, the expense and expertise these require are limiting. OCT researchers began to show an interest in SC generation after 2001 when ultrahigh resolution OCT (resolution of $2.5 \mu \mathrm{m}$ ) at $1.3 \mu \mathrm{m}$ central wavelength was demonstrated from a SC source built using microstructured fibers pumped with a mode-locked Ti:sapphire laser [21]. Nowadays, SC radiation optimized for OCT is available even from compact commercial sources using fiber and microchip lasers which can inexpensively provide a broader spectral coverage than Ti:Sapphire lasers. These new SC sources cover several interesting spectral bands like the visible range for maximum biological chromophore absorption and sub-micrometer ultra-high resolution OCT, the $1.05 \mu \mathrm{m}$ range for ophthalmic OCT with enhanced penetration depth and reduced scattering, and the 1.3-1.7 $\mu \mathrm{m}$ range for in vivo non-invasive biopsies [83]. Additionally, the broad bandwidth can be exploited to perform spectroscopic OCT. However, ultra-broad SC generated from an interplay of various nonlinear effects in the ADi regime still suffer from instability (shot-to-shot variations) and uneven (non-Gaussian) spectral profile, leading to multiple im- age echoes hiding weak object structures and reducing the resolution and sensitivity. Pulse shaping implementations have been used to create Gaussian spectra but this comes with large losses in output power. A noteworthy attempt to reduce noise while simultaneously shaping the pulse without much loss has used appropriate tapering of PCFs to manipulate fiber dispersion to be in the NDi regime for all wavelengths, eliminating modulation instability and soliton effects [84]. Going forward, improving the stability of SC generated from longer pump pulses is the key to compact, cost-effective yet high-performance OCT.

\section{F. Coherent Anti-Stokes Raman Scattering (CARS) mi- croscopy}

Other useful biological imaging applications of SC sources are CARS microscopy and CARS microspectroscopy - techniques allowing visualization of chemical composition and structures of samples, based on their characteristic intrinsic vibrational contrast. CARS is label-free and has a non-deteriorating signal, unlike in fluorescence microscopy where photobleaching eventually causes a large reduction in the SNR. CARS microscopy requires two beams (a pump pulse and a synchronous broadband Stokes pulse) and produces a signal when the difference between pump and Stokes frequencies matches the Raman resonance frequency of the sample [86]. These synchronous beams are difficult to produce without using two synchronized lasers or complex setups involving OPA and OPOs, or four-wave-mixingbased sources which are the current state-of-the-art methodologies. However, these beams are also readily produced through SC generation [87] using fs lasers pumping PCFs tailored to produce appropriate wavelengths. Temporally coherent SC radiation has so far only provided low peak powers and low spectral power density. For a reasonably high-resolution CARS image, this poses a severe limitation of long acquisition times [88].

However, other techniques like multiplex CARS do not require high temporal coherence. Incoherent and ultra-broadband SC generated in the ADi regime using longer pulses and greater peak powers is ideally suited for multiplex CARS, especially when multiple signals can be generated and detected hyperspectrally [89]. Multiplex CARS microscopy with sub-micrometer resolution has been demonstrated [90] from broadband SC $\left(>2000 \mathrm{~cm}^{-1}\right)$, combined with TPE of fluorescence [86], and with second harmonic generation (SHG) and third harmonic generation (THG) signals [85] to study proteins and lipids in cells (see Figure 8 ). This multiplexed nonlinear approach permits differ- 


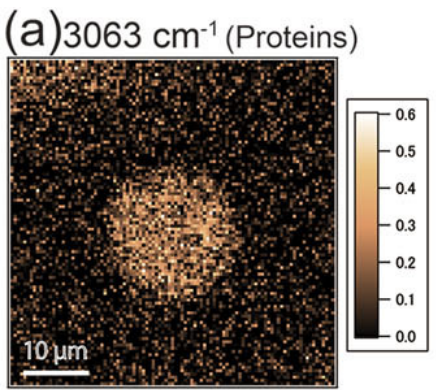

(e) $1738 \mathrm{~cm}^{-1}$ (Lipids)
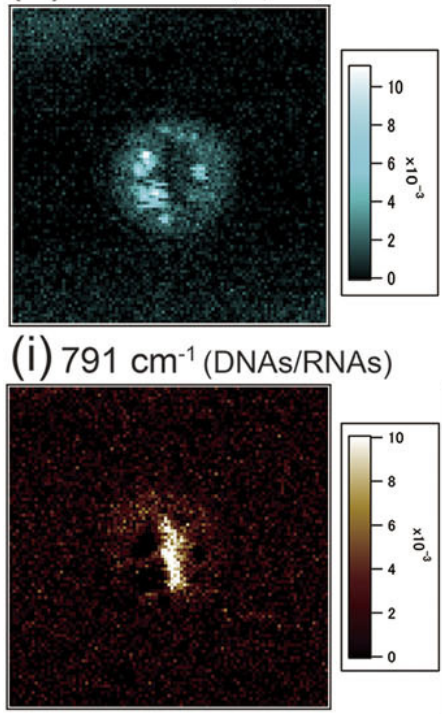

(b) $3010 \mathrm{~cm}^{-1}$ (Lipids)

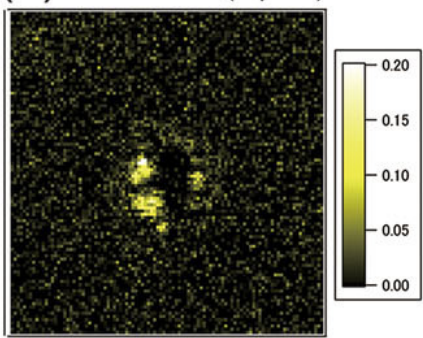

(f) $1574 \mathrm{~cm}^{-1}$ (DNAs/RNAs)

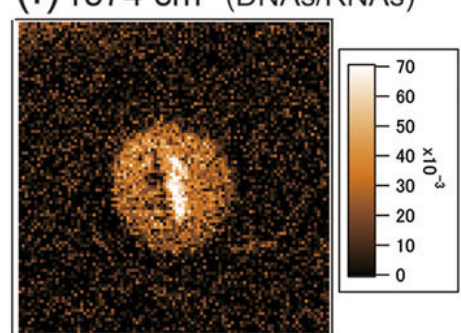

(j) $719 \mathrm{~cm}^{-1}$ (Lipids(choline))

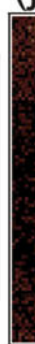

(c) $2930 \mathrm{~cm}^{-1}$ (Proteins/Lipids)
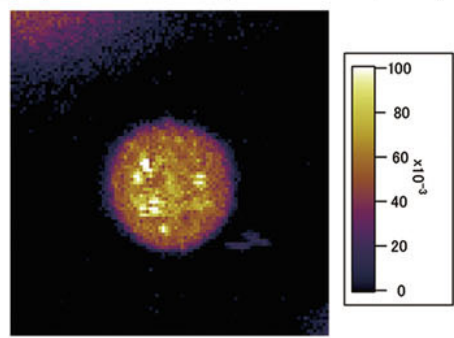

(g) $1098 \mathrm{~cm}^{-1}$ (DNAs/RNAs)

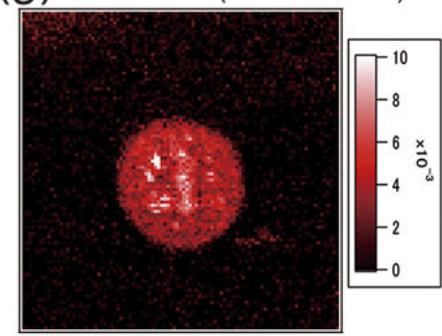

(k) SHG
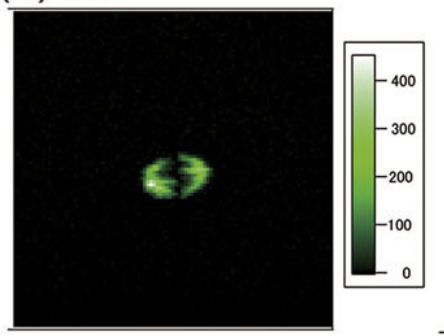

(d) $2851 \mathrm{~cm}^{-1}$ (Lipids)

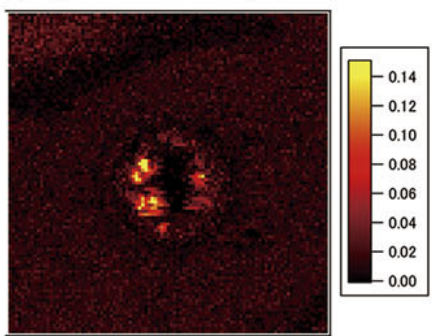

(h) $1004 \mathrm{~cm}^{-1}$ (Proteins)

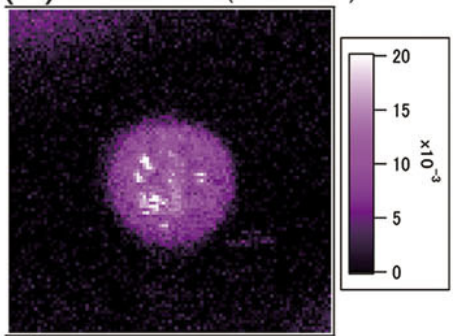

(I)THG

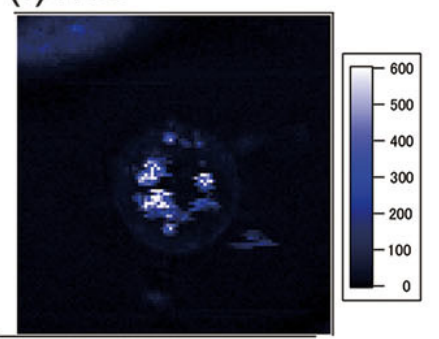

Fig. 8. CARS, second and third harmonic generation images of a HeLa cell in the mitotic phase. Chromosomes appear in a line in the center of the cell in the DNA/RNA images and spindle fibers are seen in the SHG images. CARS images at (a-j) 3063, 3010, 2930, 2851, 1738, 1574, 1098, 1004, 791, and $719 \mathrm{~cm}^{-1}$, and the (k) SHG and (l) THG images. Adapted from [85].

ent types of contrast to be achieved simultaneously to study dynamics of living cellular structures. Even compact, turn-key solutions are commercially available now that use IR SC [22] for such multi-modal imaging.

\section{CHALLENGES AND RECENT ADVANCEMENTS}

It is clear from the various applications mentioned above that the useful features of compact SC sources like spectral flexibility, moderately high spectral power density, fast pulses, low cost, low maintenance and small footprint have had a significant impact in biological and clinical imaging research. Despite their growing popularity, making SC more reliable and universal in imaging applications demands overcoming some important technological challenges. We briefly discuss these below. PCF parameters largely determine the underlying spectral broadening and SC stability so better PCF design and simulation, and their reproducible manufacturing with good fiber quality is at the heart of tackling many of these challenges.

\section{A. UV generation}

There is great demand to expand the coverage of SC sources to the UV range from 200-400nm and a number of recent efforts have been made in this context [92]. UV coverage would find broad applications in fluorescence microscopy, spectroscopy and biomedical photonics since many molecules of interest important in cancer and metabolism research, like NADH and FAD, have an intrinsic fluorescence signature when excited by UV light. One scheme to reach UV uses generation of second-, thirdand fourth- harmonic of mode-locked Ti: $\mathrm{Al}_{2} \mathrm{O}_{3}$ laser to go down to $205 \mathrm{~nm}$ but has very complex and limited tuning capability [93]. If an optical parametric oscillator is used in conjunction, the tunability can be expanded from 250-355nm [94] but the very high system complexity makes it impractical for general microscopy use. Another technique uses SC generation assisted by cascaded four-wave mixing in a PCF to cover the entire UV-A range $(300-400 \mathrm{~nm})$ [22]. The issue with SC generation in the UV using popular silica based PCFs is that they undergo UV damage (solarization) with wavelengths under $380 \mathrm{~nm}$. There have been a few noteworthy advances in the last few years to replace silica in PCFs with exotic glasses like ZBLAN [95] to increase long term viability. This allows generating SC with shorter UV wavelengths while still being ultra-broadband, spanning more than three octaves in the 200-2500nm range. Even relatively low power and compact laser pumps can be used for this. Similarly, exciting advances in gas-filled hollow-core (HC) fibers, which use photonic bandgaps for trapping and guiding light within a central hollow core [12], have allowed harnessing the UV-transparency and low UV damage susceptibility of gases. While these HC fibers usually suffer from higher propagation losses, certain microstructured designs allow low loss transmission, higher optical damage thresholds and also exquisite control of fiber nonlinearity and group-velocity dispersion (GVD), with 


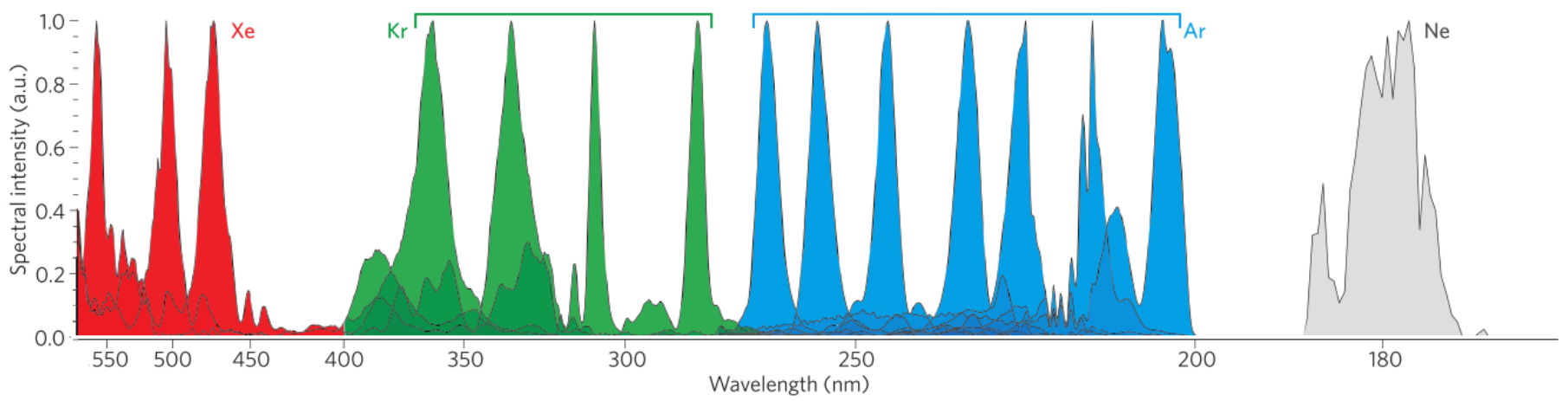

Fig. 9. Coherent ultrashort pulses of tunable bandwidths in the UV range generated in gas-filled hollow-core PCFs, by adjusting pressure and gas mixtures ( $\mathrm{Ne}, \mathrm{Ar}, \mathrm{Kr}, \mathrm{Xe}$ gases). Adapted from [91] and [12].

a smooth and broadband ADi profile simply by varying pressure and gas mixtures $[96,97]$. The normal GVD of a chosen noble gas can be balanced against the anomalous GVD of the kagome PCF to tune the ZDW across NIR, visible, and even in the UV. With this level of control, the tunable wavelength can be pushed into the deep-UV and also just as easily into the IR up to $2500 \mathrm{~nm}$, using a relatively low power and compact laser pump, with wide-ranging applications in sensing and photochemistry studies [98]. The generation of a coherent, ultrafast, deep-ultraviolet signal, optimized to be emitted in a narrow wavelength band tunable from 176-550nm has been demonstrated [12] by varying pressure in inert gases like $\mathrm{Ne}, \mathrm{Ar}, \mathrm{Kr}$ and $\mathrm{Xe}$ (see Fig. 9). The same group also demonstrated the shortest optical wavelength in a SC generated to date, from 124nm-1200nm using Raman active hydrogen-filled HC PCF [99]. However, all these results from HC PCFs so far have used fs input pulses with $\mu \mathrm{J}$ pulse energies (with the exception of a recent paper [100] using 500nJ pulse energy), which sets the source requirements quite high and with very low repetition rates in the $\mathrm{kHz}$ making time-resolved imaging very slow. Less stringent source requirements would open the door for widespread use, especially if $\mathrm{MHz}$ repetition rates and high average powers could be achieved in the deep UV to IR range. Simple, compact sources of spatially coherent and ultrafast UV light with long-term stable operation would have excellent application potential in fluorescence microscopy and in the fields of UV absorption spectroscopy, UV-resonant Raman spectroscopy and laser lithography.

\section{B. Near-infrared microscopy}

On the other end of the visible spectrum is the near infra-red optical window (650-900nm), easily accessible using a SC source and silicon based detectors (for $\lambda<1000 \mathrm{~nm}$ ) with great potential for in-vivo optical imaging of deep tissues. This $650-900 \mathrm{~nm}$ window has minimized light-scattering and therefore reduced blur, as well as minimal absorbance by hemoglobin, water and lipids [101], allowing more photons to reach the detector. Genetically encoded infra-red fluorescent proteins would be particularly valuable for whole-body imaging in cancer, stem cell biology, gene therapy, etc. However, the challenges in realizing this opportunity has less to do with SC improvements and more with accessibility and availability of good fluorescent proteins in this range. Over the last few years, the toolbox of fluorescent proteins engineered to push the spectrum further into the IR has been growing [102, 103]. Future work targeting improvements in long-term biocompatibility, photostability and brightness to match the characteristics of widely used fluorophores like EGFP will surely open new vistas for in-vivo deep tissue imaging in animals. Longer NIR wavelengths have so far been overlooked for imaging due to the lack of appropriate laser sources and suitable detectors. However, with a SC source and the arrival of new IR-CCD photodetectors such as indium gallium arsenide (InGaAs, $\lambda$ upto 1700nm) and indium antimonide (InSb, for $\lambda>1700$ ) based detectors, novel imaging opportunities can be realized. Recent research[50] utilizing SC has identified new optical windows (II: 1100-1350nm, III: 1600-1870nm and IV: centered at 2200nm) with better transmission, minimal absorption and reduced scattering than the optical window I (650-900nm) for deep brain imaging [104].

\section{On-chip supercontinuum generation}

SC generation has already been demonstrated in various chipbased waveguides. These can become important elements to expand the functionality of photonic integrated circuits or be useful in integrated chip-based imaging applications in the future. SC has been generated in silicon photonic nanowires [105], in silica [106] and chalcogenide [107] waveguides although only silicon nitride $[108,109]$ waveguides have allowed pushing the spectrum to broaden towards visible wavelengths for potential on-chip imaging applications.

\section{Stability with greater output powers}

Increasing SC stability while at the same time increasing spectral power density with watt-level total output and broad bandwidth has always been one of the big technological challenges in the SC field. While each of these are easily possible separately (former in fs NDi regime and latter using continuous waves), combining these two output characteristics using the same pump source has proven to be very difficult, if not impossible due to the physical mechanisms governing SC generation. Instability in the fs ADi regime has previously been tackled through an alternative approach to solid core silica PCFs - by using tapered fibers that allow large freedom in controlling the dispersion profile. In the demonstrated tapered fiber [84] tailored to obtain smooth spectra for OCT applications, initial broadening occurs efficiently in the ADi regime, and then evolves into NDi broadening along the taper, resulting in smoother spectra and improved stability due to the NDi. Despite the stability improvement, this approach is not very useful for obtaining greater output powers.

If higher peak powers in coherent SC from fs pump sources are desired, HC kagome PCFs can again prove to be useful. They can accommodate extremely large pulse energies and higher peak-power fs pulses in the fiber, unlike solid core PCFs which 
would undergo optical damage [99]. Recent advances in gasfilled HC-PCFs with versatile control of dispersion and nonlinearity can allow generating more stable SC [100]. Numerical simulations have shown that the generated dispersive wave pulses in these fibers are highly coherent and independent of input pulse energy when the pulse durations are tens of fs [91]. However, the bigger problem of achieving higher stability with longer pump pulses still exists. Future developments to resolve this issue will enable a huge range of applications.

\section{CONCLUSIONS}

SC sources provide spectral versatility and spatially coherent, high brightness radiation often at affordable costs and smaller footprint than established tunable laser technologies. The features offered by the compact pulsed SC laser make it an ideal source for a wide range of biophotonics applications in fluorescence microscopy and biomedical imaging. Recent advances in novel PCF-based technologies and hollow-core fibers offer promise that current challenges will be surmounted in the near future, and that the use of SC sources will become commonplace, replacing many monochromatic and complex tunable laser sources in research laboratories and in the clinic.

\section{FUNDING}

This project has received funding from the European Union's H2020-MSCA-ITN-2016 research and innovation programme under the Marie Sklodowska-Curie Grant Agreement No. 722380 (SUPUVIR). CFK acknowledges funding from the UK Engineering and Physical Sciences Research Council, EPSRC (grants $\mathrm{EP} / \mathrm{L} 015889 / 1$ and EP/H018301/1), the Wellcome Trust (grants 3-3249/Z/16/Z and 089703/Z/09/Z), the UK Medical Research Council, MRC (grants MR/K015850/1 and MR/K02292X/1), MedImmune, and Infinitus (China), Ltd.

\section{REFERENCES}

1. S. W. Hell and J. Wichmann, "Breaking the Diffraction Resolution Limit By Stimulated-Emission - Stimulated-Emission-Depletion Fluorescence Microscopy," Opt. Lett. 19, 780-782 (1994).

2. M. G. L. Gustafsson, "Surpassing the lateral resolution limit by a factor of two using structured illumination microscopy," J. Microsc. 198, 82-87 (2000).

3. E. Betzig, G. H. Patterson, R. Sougrat, O. W. Lindwasser, S. Olenych, J. S. Bonifacino, M. W. Davidson, J. Lippincott-schwartz, and H. F. Hess, "Imaging Intracellular Fluorescent Proteins at Nanometer Resolution," Science. 313, 1642-1646 (2006).

4. M. Rust, M. Bates, and X. Zhuang, "Stochastic optical reconstruction miscroscopy (STORM) provides sub-diffraction-limit image resolution," Nat. Methods 3, 793-795 (2006).

5. W. Becker, "Fluorescence lifetime imaging - techniques and applications," J. Microsc. 247, 119-136 (2012).

6. G. Genty, S. Coen, and J. M. Dudley, "Fiber supercontinuum sources (Invited)," J. Opt. Soc. Am. B 24, 1771 (2007).

7. J. M. Dudley, G. Genty, and S. Coen, "Supercontinuum generation in photonic crystal fiber," Rev. Mod. Phys. 78, 1135-1184 (2006).

8. P. S. J. Russell, "Photonic crystal fibers." Science. 299, 358-362 (2003).

9. J. Knight, "Photonic crystal fibres," Nature. 424, 847-851 (2003).

10. J. R. Taylor, "Introduction and history," in Supercontinuum Generation in Optical Fibers, J. M. Dudley and J. R. Taylor, eds. (Cambridge University Press, Cambridge, 2010), pp. 1-29.

11. R. R. Alfano, ed., The Supercontinuum Laser Source (Springer-Verlag, New York, 2006).
12. P. S. J. Russell, P. Hölzer, W. Chang, A. Abdolvand, and J. C. Travers, "Hollow-core photonic crystal fibres for gas-based nonlinear optics," Nat. Photonics 8, 278-286 (2014).

13. W. Belardi and J. C. Knight, "Hollow antiresonant fibers with reduced attenuation," Opt. Lett. 39, 1853-1856 (2014).

14. R. Sollapur, D. Kartashov, M. Zürch, A. Hoffmann, T. Grigorova, G. Sauer, A. Hartung, A. Schwuchow, J. Bierlich, J. Kobelke, M. Chemnitz, M. A. Schmidt, and C. Spielmann, "Resonance-enhanced multioctave supercontinuum generation in antiresonant hollow-core fibers," Light. Sci. \& Appl. 6, e17124 (2017).

15. R. S. Watt, C. F. Kaminski, and J. Hult, "Generation of supercontinuum radiation in conventional single-mode fibre and its application to broadband absorption spectroscopy," Appl. Phys. B: Lasers Opt. 90, 47-53 (2008).

16. J. Hult, R. S. Watt, and C. F. Kaminski, "High bandwidth absorption spectroscopy with a dispersed supercontinuum source," Opt. Express 15, 11385 (2007).

17. J. M. Langridge, T. Laurila, R. S. Watt, R. L. Jones, C. F. Kaminski, and J. Hult, "Cavity enhanced absorption spectroscopy of multiple trace gas species using a supercontinuum radiation source." Opt. express 16, 10178-10188 (2008).

18. C. F. Kaminski, J. Hult, R. S. Watt, and T. Laurila, "Cavity Enhanced Spectroscopy of High-Temperature $\mathrm{H}<\mathrm{sub}>2</ \mathrm{sub}>\mathrm{O}$ in the NearInfrared Using a Supercontinuum Light Source," Appl. Spectrosc. Vol. 63, Issue 12, pp. 1389-1395 63, 1389-1395 (2009).

19. T. Udem, R. Holzwarth, and T. W. Hänsch, "Optical frequency metrology," Nature 416, 233-237 (2002).

20. J. Kasparian, M. Rodriguez, G. Mejean, J. Yu, E. Salmon, H. Wille, R. Bourayou, S. Frey, Y. B. Andre, A. Mysyrowicz, R. Sauerbrey, J. P. Wolf, and L. Woste, "White-light filaments for atmospheric analysis," Science 301, 61-64 (2003).

21. I. Hartl, X. D. Li, C. Chudoba, R. K. Ghanta, T. H. Ko, J. G. Fujimoto, J. K. Ranka, and R. S. Windeler, "Ultrahigh-resolution optical coherence tomography using continuum generation in an air-silica microstructure optical fiber," Opt. Lett. 26, 608-610 (2001).

22. A. Labruyère, A. Tonello, V. Couderc, G. Huss, and P. Leproux, "Compact supercontinuum sources and their biomedical applications," Opt. Fiber Technol. 18, 375-378 (2012).

23. C. F. Kaminski, R. S. Watt, A. D. Elder, J. H. Frank, and J. Hult, "Supercontinuum radiation for applications in chemical sensing and microscopy," Appl. Phys. B: Lasers Opt. 92, 367-378 (2008).

24. S. Dupont, Z. Qu, S. S. Kiwanuka, L. E. Hooper, J. C. Knight, S. R. Keiding, and C. F. Kaminski, "Ultra-high repetition rate absorption spectroscopy with low noise supercontinuum radiation generated in an all-normal dispersion fibre," Laser Phys. Lett. 11, 75601 (2014).

25. J. K. Ranka, R. S. Windeler, and A. J. Stentz, "Visible continuum generation in air-silica microstructure optical fibers with anomalous dispersion at $800 \mathrm{~nm}$," Opt. Lett. 25, 25 (2000).

26. T. A. Birks, W. J. Wadsworth, and P. S. J. Russell, "Supercontinuum generation in tapered fibers," Opt. Lett. 25, 1415 (2000).

27. W. Wadsworth, N. Joly, J. Knight, T. Birks, F. Biancalana, and P. Russell, "Supercontinuum and four-wave mixing with Q-switched pulses in endlessly single-mode photonic crystal fibres." Opt. express 12, 299-309 (2004).

28. G. P. Agrawal, Nonlinear fiber optics (Academic Press, 2013).

29. A. V. Husakou and J. Herrmann, "Supercontinuum generation of higherorder solitons by fission in photonic crystal fibers." Phys. Rev. Lett. 87, 203901 (2001).

30. J. Dudley and J. Taylor, Supercontinuum Generation in Optical Fibers (Cambridge University Press, 2010).

31. G. Mcconnell, "Confocal laser scanning fluorescence microscopy with a visible continuum source," Opt. Express 12, 2844-2850 (2004).

32. C. Dunsby, P. M. P. Lanigan, J. McGinty, D. S. Elson, J. Requejo-Isidro, I. Munro, N. Galletly, F. McCann, B. Treanor, B. Önfelt, D. M. Davis, M. a. a. Neil, and P. M. W. French, "An electronically tunable ultrafast laser source applied to fluorescence imaging and fluorescence lifetime imaging microscopy," J. Phys. D: Appl. Phys. 37, 3296-3303 (2004).

33. J. A. Palero, V. O. Boer, J. C. Vijverberg, H. C. Gerritsen, and H. J. C. M. 
Sterenborg, "Short-wavelength two-photon excitation fluorescence microscopy of tryptophan with a photonic crystal fiber based light source." Opt. express 13, 5363-8 (2005).

34. T. Betz, J. Teipel, D. Koch, W. Härtig, J. Guck, J. Käs, and H. Giessen, "Excitation beyond the monochromatic laser limit: simultaneous 3-D confocal and multiphoton microscopy with a tapered fiber as white-light laser source." J. biomedical optics 10, 054009 (2005).

35. M. Klimczak, G. Soboń, R. Kasztelanic, K. M. Abramski, and R. Buczyński, "Direct comparison of shot-to-shot noise performance of all normal dispersion and anomalous dispersion supercontinuum pumped with sub-picosecond pulse fiber-based laser," Sci. Reports $\mathbf{6}$, 19284 (2016).

36. J. M. Dudley, G. Genty, and S. Coen, "Fibre supercontinuum generation overview," in Supercontinuum Generation in Optical Fibers, J. M. Dudley and J. R. Taylor, eds. (Cambridge University Press, Cambridge, 2010), pp. 52-61.

37. C. Dunsby and P. M. W. French, "Biophotonics applications of supercontinuum generation," in Supercontinuum Generation in Optical Fibers, J. M. Dudley and J. R. Taylor, eds. (Cambridge University Press, Cambridge, 2010), pp. 349-372.

38. J. R. Clowes, A. B. Grudinin, and I. M. Godfrey, "FLEXIBLE AND STABLE SUPERCONTINUUM SOURCE," (2015)

39. K. K. Chen, S.-u. Alam, J. H. V. Price, J. R. Hayes, D. Lin, A. Malinowski, C. Codemard, D. Ghosh, M. Pal, S. K. Bhadra, and D. J. Richardson, "Picosecond fiber MOPA pumped supercontinuum source with $39 \mathrm{~W}$ output power," Opt. Express 18, 5426-5432 (2010).

40. J. C. Travers, A. B. Rulkov, B. A. Cumberland, S. V. Popov, and J. R. Taylor, "Visible supercontinuum generation in photonic crystal fibers with a 400W continuous wave fiber laser," Opt. Express 16, 1443514447 (2008).

41. A. Mussot and A. Kudlinski, "19.5 W CW-pumped supercontinuum source from 0.65 to $1.38 \mu \mathrm{m}$," Electron. Lett. 45, 29 (2009).

42. A. Kudlinski and A. Mussot, "Optimization of continuous-wave supercontinuum generation," Opt. Fiber Technol. 18, 322-326 (2012).

43. R. Mercatelli, S. Soria, G. Molesini, F. Bianco, G. Righini, and F. Quercioli, "Supercontinuum source tuned by an on-axis monochromator for fluorescence lifetime imaging." Opt. express 18, 20505-11 (2010).

44. P. E. Buchsbaum and J. D. Lane, "United States Patent US6700690 B1- TUNABLE VARIABLE BANDPASS OPTICAL FILTER," (2004).

45. R. Borlinghaus, H. Gugel, P. Albertano, and V. Seyfried, "Closing the spectral gap: The transition from fixed-parameter fluorescence to tunable devices in confocal microscopy," Proc. SPIE 6090, 60900T-6 (2006).

46. H. Birk, J. Engelhardt, R. Storz, N. Hartmann, J. Bradl, H. Ulrich, L. Microsystems, H. Gmbh, A. Friedensplatz, and D. Mannheim, "Programmable beam-splitter for confocal laser scanning microscopy," Proc. SPIE 4621, 16-27 (2002).

47. A. Periasamy, P. Wodnicki, X. F. Wang, S. Kwon, G. W. Gordon, and B. Herman, "Time-resolved fluorescence lifetime imaging microscopy using a picosecond pulsed tunable dye laser system," Rev. Sci. Instruments 67, 3722-3731 (1996)

48. J. H. Frank, A. D. Elder, J. Swartling, A. R. Venkitaraman, A. D. Jeyasekharan, and C. F. Kaminski, "A white light confocal microscope for spectrally resolved multidimensional imaging," J. Microsc. 227, 203215 (2007).

49. S. Schlachter, A. Elder, J. H. Frank, A. Grudinin, and C. F. Kaminski, "Spectrally Resolved Confocal Fluorescence Microscopy with a Supercontinuum Laser," Microsc. Analysis 22, 11-13 (2008).

50. L. A. Sordillo, L. Lindwasser, Y. Budansky, P. Leproux, and R. R. Alfano, "Near-infrared supercontinuum laser beam source in the second and third near-infrared optical windows used to image more deeply through thick tissue as compared with images from a lamp source," J. Biomed. Opt. 20, 030501 (2015).

51. B. Redding, M. A. Choma, and H. Cao, "Speckle-free laser imaging using random laser illumination," Nat. Photonics 6, 355-359 (2012).

52. P. Georgiades, V. J. Allan, M. Dickinson, and T. A. Waigh, "Reduction of coherent artefacts in super-resolution fluorescence localisation microscopy," J. Microsc. 264, 375-383 (2016).
53. K. Shi, P. Li, S. Yin, and Z. Liu, "Chromatic confocal microscopy using supercontinuum light." Opt. express 12, 2096-2101 (2004).

54. C. Olsovsky, R. Shelton, O. Carrasco-Zevallos, B. E. Applegate, and K. C. Maitland, "Chromatic confocal microscopy for multi-depth imaging of epithelial tissue." Biomed. optics express 4, 732-40 (2013).

55. A. Esposito, A. N. Bader, S. C. Schlachter, D. J. V. D. H, G. S. K. Schierle, R. V. Ashok, C. F. K, and H. C. Ger, "Design and application of a confocal microscope for spectrally resolved anisotropy imaging," Opt. Express 19, 2546-2555 (2011).

56. D. M. Owen, E. Auksorius, H. B. Manning, C. B. Talbot, P. a. a. de Beule, C. Dunsby, M. a. a. Neil, and P. M. W. French, "Excitation-resolved hyperspectral fluorescence lifetime imaging using a UV-extended supercontinuum source." Opt. letters 32, 3408-3410 (2007).

57. T. Zimmermann, J. Rietdorf, and R. Pepperkok, "Spectral imaging and its applications in live cell microscopy," FEBS Lett. 546, 87-92 (2003).

58. M. Dickinson, G. Bearman, S. Tille, R. Lansford, and S. E. Fraser, "Multi-spectral imaging and linear unmixing add a whole new dimension to laser scanning flourescence microscopy," Bioimaging. 31, 1272 1278 (2001).

59. G. Lu and B. Fei, "Medical hyperspectral imaging: a review." J. biomedical optics 19, 10901 (2014).

60. V. V. Tuchin, Tissue Optics: Light Scattering Methods and Instruments for Medical Diagnosis (SPIE Press, 2015).

61. K. Suhling, L. M. Hirvonen, J. a. Levitt, P.-H. Chung, C. Tregidgo, A. Le Marois, D. a. Rusakov, K. Zheng, S. Ameer-Beg, S. Poland, S. Coelho, R. Henderson, and N. Krstajic, "Fluorescence lifetime imaging (FLIM): Basic concepts and some recent developments," Med. Photonics 44, 3-40 (2015).

62. H. B. Manning, G. T. Kennedy, D. M. Owen, D. M. Grant, A. I. Magee, M. A. A. Neil, Y. Itoh, C. Dunsby, and P. M. W. French, "A compact, multidimensional spectrofluorometer exploiting supercontinuum generation," J. Biophotonics 1, 494-505 (2008).

63. D. M. Grant, D. S. Elson, D. Schimpf, C. Dunsby, E. Auksorius, I. Munro, M. A. A. Neil, P. M. W. French, E. Nye, G. Stamp, and P. Courtney, "Optically sectioned fluorescence lifetime imaging using a Nipkow disk microscope and a tunable ultrafast continuum excitation source," Opt. letters 30, 3353-3355 (2005)

64. P. Blandin, S. Lévêque-Fort, S. Lécart, J. C. Cossec, M.-C. Potier, Z. Lenkei, F. Druon, and P. Georges, "Time-gated total internal reflection fluorescence microscopy with a supercontinuum excitation source." Appl. optics 48, 553-559 (2009).

65. A. D. Elder, C. F. Kaminski, and J. H. Frank, "phi2-FLIM: a technique for alias-free frequency domain fluorescence lifetime imaging," Opt. Express 17, 23181-23203 (2009).

66. S. Schlachter, A. D. Elder, A. Esposito, G. S. Kaminski, J. H. Frank, L. K. van Geest, and C. F. Kaminski, "mhFLIM: resolution of heterogeneous fluorescence decays in widefield lifetime microscopy." Opt. express 17 , 1557-70 (2009).

67. Schlachter, Schwedler, Esposito, Schierle, Moggridge, and Kaminski, "A method to unmix multiple fluorophores in microscopy images with minimal a priori information," Opt. Express 17, 22747-22760 (2009).

68. D. M. Grant, J. Mcginty, E. J. Mcghee, T. D. Bunney, D. M. Owen, C. B. Talbot, W. Zhang, S. Kumar, I. Munro, P. M. P. Lanigan, G. T. Kennedy, C. Dunsby, A. I. Magee, P. Courtney, M. Katan, M. A. A. Neil, and P. M. W. French, "High speed optically sectioned fluorescence lifetime imaging permits study of live cell signaling events," Opt. Express 15, 15656-15673 (2007).

69. W. Chen, E. Avezov, S. C. Schlachter, F. Gielen, R. F. Laine, H. P. Harding, F. Hollfelder, D. Ron, and C. F. Kaminski, "Biophysical Letter A Method to Quantify FRET Stoichiometry with Phasor Plot Analysis and Acceptor Lifetime Ingrowth," Biophysj 108, 999-1002 (2015).

70. T. Dertinger and S. Rüttinger, "Advanced FCS: An Introduction to Fluorescence Lifetime Correlation Spectroscopy and Dual-Focus FCS," in Advanced Photon Counting, (Springer, Cham, 2014), pp. 89-109.

71. M. Fernandez-Suarez and A. Y. Ting, "Fluorescent probes for superresolution imaging in living cells," Nat Rev Mol Cell Biol 9, 929-943 (2008).

72. D. Wildanger, E. Rittweger, L. Kastrup, and S. W. Hell, "STED mi- 
croscopy with a supercontinuum laser source," Opt. Express 110 , 1726-1734 (2008).

73. M. D. Lesoine, S. Bose, J. W. Petrich, and E. A. Smith, "Supercontinuum stimulated emission depletion fluorescence lifetime imaging," J. Phys. Chem. B 116, 7821-7826 (2012).

74. C. Osseforth, J. R. Moffitt, L. Schermelleh, and J. Michaelis, "Simultaneous dual-color 3D STED microscopy." Opt. express 22, 7028-39 (2014).

75. C. Lefort, R. P. O. Connor, V. Blanquet, L. Magnol, H. Kano, V. Tombelaine, P. Lévêque, V. Couderc, and P. Leproux, "Multicolor multiphoton microscopy based on a nanosecond supercontinuum laser source," J. Biophotonics 714, 709-714 (2016).

76. J. Yi, W. Liu, S. Chen, V. Backman, N. Sheibani, C. M. Sorenson, A. A. Fawzi, R. A. Linsenmeier, and H. F. Zhang, "Visible light optical coherence tomography measures retinal oxygen metabolic response to systemic oxygenation," Light. Sci. \& Appl. 4, e334 (2015).

77. F. Helmchen and W. Denk, "Deep tissue two-photon microscopy," Nat. Methods 2, 932-940 (2005).

78. E. E. Hoover and J. A. Squier, "Advances in multiphoton microscopy technology," Nat. Photonics 7, 93-101 (2013).

79. G. McConnell and E. Riis, "Photonic crystal fibre enables shortwavelength two-photon laser scanning fluorescence microscopy with fura-2," Phys. Medicine Biol. 49, 4757-4763 (2004).

80. K. Isobe, W. Watanabe, S. Matsunaga, T. Higashi, K. Fukui, and K. Itoh, "Multi-spectral two-photon excited fluorescence microscopy using supercontinuum light source," Jpn. J. Appl. Physics, Part 2: Lett. 44 (2005).

81. R. A. Costa, M. Skaf, L. A. S. Melo, D. Calucci, J. A. Cardillo, J. C. Castro, D. Huang, and M. Wojtkowski, "Retinal assessment using optical coherence tomography," Prog. Retin. Eye Res. 25, 325-353 (2006).

82. Z. Zhi, J. Qin, L. An, and R. K. Wang, "Supercontinuum light source enables in vivo optical microangiography of capillary vessels within tissue beds." Opt. letters 36, 3169-71 (2011).

83. A. Unterhuber, B. Povazay, A. Aguirre, Y. Chen, F. X. Kärtner, J. G. Fujimoto, and W. Drexler, "Broad Bandwidth Laser and Nonlinear Optical Light Sources for OCT," in Optical Coherence Tomography, (Springer, Berlin, Heidelberg, 2008), pp. 301-358.

84. P. Falk, M. H. Frosz, O. Bang, H. Morioka, S. Takara, O. Kawanishi, K. Kamatani, K. Takiguchi, M. Uchiyama, H. Saruwatari, M. Takahashi, T. Yamada, and H. Kanamori, "Supercontinuum generation in a photonic crystal fiber with two zero-dispersion wavelengths tapered to normal dispersion at all wavelengths," Opt. Express 13, 7535-7540 (2005).

85. H. Yoneyama, K. Sudo, P. Leproux, V. Couderc, A. Inoko, and H. Kano, "Invited Article: CARS molecular fingerprinting using sub-100-ps microchip laser source with fiber amplifier," APL Photonics 3 (2018).

86. H. Kano and H.-o. Hamaguchi, "Supercontinuum Dynamically Visualizes a Dividing Single Cell," Anal. Chem. 79, 8967-8973 (2007).

87. P. Klarskov, A. Isomäki, K. P. Hansen, and P. E. Andersen, "Supercontinuum generation for coherent anti-Stokes Raman scattering microscopy with photonic crystal fibers," Opt. Express 19, 26672 (2011).

88. T. Gottschall, T. Meyer, M. Baumgartl, C. Jauregui, M. Schmitt, J. Popp, J. Limpert, and A. Tünnermann, "Fiber-based light sources for biomedical applications of coherent anti-Stokes Raman scattering microscopy," Laser \& Photonics Rev. 9, 435-451 (2015).

89. H. Kano, H. Segawa, M. Okuno, P. Leproux, and V. Couderc, "Hyperspectral coherent Raman imaging - Principle, theory, instrumentation, and applications to life sciences," J. Raman Spectrosc. 47, 116-123 (2016).

90. M. Okuno, H. Kano, P. Leproux, V. Couderc, and H. Hamaguchi, "Ultrabroadband multiplex CARS microspectroscopy and imaging using a subnanosecond supercontinuum light source in the deep near infrared," Opt. Lett. 33, 923-925 (2008).

91. K. F. Mak, J. C. Travers, P. Hölzer, N. Y. Joly, and P. S. J. Russell, "Tunable vacuum-UV to visible ultrafast pulse source based on gasfilled Kagome-PCF," Opt. Express 21, 10942 (2013).

92. J. C. Travers, T. F. Grigorova, and F. Belli, "Ultraviolet Supercontinuum
Generation in Optical Fibers," in Conference on Lasers and ElectroOptics, (OSA, Washington, D.C., 2018), p. ATu3S.2.

93. A. Nebel and R. Beigang, "External frequency conversion of cw modelocked Ti:Al2O3 laser radiation," Opt. Lett. 16, 1729-1731 (1991).

94. M. Ghotbi, A. Esteban-Martin, and E.-Z. M, "Tunable high-repetitionrate femtosecond pulse generation in the ultraviolet," Opt. Lett. 33, 345-347 (2008).

95. X. Jiang, N. Y. Joly, M. A. Finger, F. Babic, G. K. L. Wong, J. C. Travers, and P. S. J. Russell, "Deep-ultraviolet to mid-infrared supercontinuum generated in solid-core ZBLAN photonic crystal fibre," Nat. Photonics 9, 133-139 (2015)

96. N. Y. Joly, J. Nold, W. Chang, P. Holzer, A. Nazarkin, G. K. L. Wong, F. Biancalana, and P. S. J. Russell, "Bright spatially coherent wavelength-tunable deep-UV laser source using an Ar-filled photonic crystal fiber," Phys. Rev. Lett. 106, 1-4 (2011).

97. J. C. Travers, W. Chang, J. Nold, N. Y. Joly, and P. St. J. Russell, "Ultrafast nonlinear optics in gas-filled hollow-core photonic crystal fibers [Invited]," J. Opt. Soc. Am. B 28, A11 (2011).

98. A. M. Cubillas, S. Unterkofler, T. G. Euser, B. J. M. Etzold, A. C. Jones, P. J. Sadler, P. Wasserscheid, and P. S. J. Russell, "Photonic crystal fibres for chemical sensing and photochemistry," ChemSocRev 42 (2013).

99. F. Belli, A. Abdolvand, W. Chang, J. C. Travers, and P. S. Russell, "Vacuum-ultraviolet to infrared supercontinuum in hydrogen-filled photonic crystal fiber," Optica 2, 292 (2015).

100. P. Hosseini, A. Ermolov, F. Tani, D. Novoa, and P. S. J. Russell, "UV Soliton Dynamics and Raman-Enhanced Supercontinuum Generation in Photonic Crystal Fiber," ACS Photonics 5, 2426-2430 (2018).

101. X. Shu, A. Royant, M. Z. Lin, T. A. Aguilera, V. Lev-Ram, P. A. Steinbach, and R. Y. Tsien, "Mammalian Expression of Infrared Fluorescent Proteins Engineered from a Bacterial Phytochrome," Science 324 (2009).

102. E. A. Rodriguez, R. E. Campbell, J. Y. Lin, M. Z. Lin, A. Miyawaki, A. E. Palmer, X. Shu, J. Zhang, and R. Y. Tsien, "The Growing and Glowing Toolbox of Fluorescent and Photoactive Proteins," Trends Biochem. Sci. 42, 111-129 (2017).

103. G. Hong, A. L. Antaris, and H. Dai, "Near-infrared fluorophores for biomedical imaging," Nat. Biomed. Eng. 1 (2017).

104. L. Shi, L. A. Sordillo, A. Rodríguez-Contreras, and R. Alfano, "Transmission in Near-Infrared Optical Windows for Deep Brain Imaging," J Biophotonics 3, 973-982 (2016).

105. I.-W. Hsieh, X. Chen, X. Liu, J. I. Dadap, N. C. Panoiu, C.-Y. Chou, F. Xia, W. M. Green, Y. A. Vlasov, and R. M. Osgood, "Supercontinuum generation in silicon photonic wires," Opt. Express 15, 15242 (2007).

106. D. Y. Oh, D. Sell, H. Lee, K. Y. Yang, S. a. Diddams, and K. J. Vahala, "Supercontinuum generation in an on-chip silica waveguide." Opt. letters 39, 1046-8 (2014).

107. M. R. Lamont, B. Luther-Davies, D.-Y. Choi, S. Madden, and B. J. Eggleton, "Supercontinuum generation in dispersion engineered highly nonlinear $(\gamma=10 / \mathrm{W} / \mathrm{m})$ As_2S_3 chalcogenide planar waveguide," Opt. Express 16, 14938 (2008).

108. R. Halir, Y. Okawachi, J. S. Levy, M. A. Foster, M. Lipson, and A. L. Gaeta, "Ultrabroadband supercontinuum generation in a CMOScompatible platform," Opt. Lett. 37, 1685 (2012).

109. J. P. Epping, T. Hellwig, M. Hoekman, R. Mateman, A. Leinse, R. G. Heideman, A. van Rees, P. J. van der Slot, C. J. Lee, C. Fallnich, and K.-J. Boller, "On-chip visible-to-infrared supercontinuum generation with more than $495 \mathrm{THz}$ spectral bandwidth," Opt. Express 23, 19596 (2015). 